\title{
Brief biopsychosocially informed education can improve insurance workers' back pain beliefs: Implications for improving claims management behaviours
}

\author{
Darren Beales ${ }^{\mathrm{a}, *}$, Tim Mitchell ${ }^{\mathrm{a}}$, Naomi Pole ${ }^{\mathrm{b}}$ and James Weir ${ }^{\mathrm{b}}$ \\ ${ }^{a}$ School of Physiotherapy and Exercise Science, Curtin University, Perth, WA, Australia \\ ${ }^{\mathrm{b}}$ CGU Insurance Limited, West Perth, WA, Australia
}

Received 1 April 2015

Accepted 3 March 2016

\begin{abstract}
.
BACKGROUND: Biopsychosocially informed education is associated with improved back pain beliefs and positive changes in health care practitioners' practice behaviours.

OBJECTIVE: Assess the effect of this type of education for insurance workers who are important non-clinical stakeholders in the rehabilitation of injured workers.

METHODS: Insurance workers operating in the Western Australian workers' compensation system underwent two, 1.5 hour sessions of biopsychosocially informed education focusing on understanding and identifying barriers to recovery of injured workers with musculoskeletal conditions. Back pain beliefs were assessed pre-education, immediately post-education and at three-month follow-up $(n=32)$. Self-reported and Injury Management Advisor-reported assessment of change in claims management behaviours were collected at the three-month follow-up.

RESULTS: There were positive changes in the Health Care Providers' Pain and Impairment Relationship Scale ( $p=0.009)$ and Back Beliefs Questionnaire $(p=0.049)$ immediately following the education that were sustained at three-month follow-up. Positive changes in claims management behaviours were supported by self-reported and Injury Management Advisor-reported data.

CONCLUSION: This study provides preliminary support that a brief biopsychosocially informed education program can positively influence insurance workers' beliefs regarding back pain, with concurrent positive changes in claims management behaviours. Further research is required to ascertain if these changes result in improved claims management outcomes.
\end{abstract}

Keywords: Workers' compensation, musculoskeletal, claims manager, injury management advisor

\section{Introduction}

Musculoskeletal conditions (injuries and pain disorders) contribute significantly to individual and societal burden both in Australia [1-3] and internationally [4-6]. Musculoskeletal conditions are the

\footnotetext{
*Address for correspondence: Dr. Darren Beales, PhD, School of Physiotherapy and Exercise Science, Curtin University, GPO Box U1987, Perth, WA 6845, Australia. Tel.: +61 89266 4644; Fax: +61 89266 3699; E-mail: D.Beales@curtin.edu.au.
}

largest contributor to lost work time in the Western Australian workers' compensation system [7]. Musculoskeletal claims of $60+$ lost work days are particularly difficult to manage, and escalating rehabilitation costs associated with these workers appears to have minimal effect on outcomes [8]. These are trends commonplace throughout Australia [9] and internationally [10].

Given this apparent lack of positive effect on worker outcomes despite increasing rehabilitation 
costs, focus on other aspects of lost time claims management warrants consideration. Insurance workers (who in Australia most frequently pertains to Case Managers and Injury Management Advisors) are key non-clinical stakeholders in lost time claims, as they play an integral role in the management of injured workers $[11,12]$. Insurance workers have input into the facilitation and coordination of injury management and rehabilitation, and therefore can indirectly influence recovery of workers with musculoskeletal conditions. Central to optimal injury management is the early identification of barriers to recovery in injured workers and the subsequent implementation of appropriate strategies to address these barriers [13]. But recent evidence suggests insurance workers may not hold beliefs aligned with best practice guidelines [14]. Additionally, there is not always consistency in the way insurance workers address barriers in injured workers' recovery [15]. Education may be a key strategy in improving insurance workers' ability to recognize and act upon barriers to recovery in injured workers $[15,16]$.

Barriers to recovery in injured workers are known to be biopsychosocially orientated [17-21]. As such, education to understand and address these barriers to recovery may benefit from being 'biopsychosocial informed'. Such education would need to cover the biomedical and pathological understanding of work injuries, as well as the contribution of psychological factors (affective and cognitive elements) and social factors (which includes work place factors such as support), and how these factors potentially contribute to ongoing work disability. Integration of the biopsychosocial model of pain is thought to be lacking in both education of health care practitioners and clinical practice [22, 23].

Beliefs of health care practitioners are known to impact decisions related to care, with practitioners exhibiting biopsychosocially orientated beliefs more likely to adhere to best-practice management guidelines [24]. Biopsychosocially informed education can successfully change the beliefs of health care practitioners [25-28] and also the general population [29], and positively influence practice behaviours $[28,30]$. Biopsychosocially informed education might also be a reasonable strategy for improving the beliefs and practice behaviours of non-clinical stakeholders (insurance workers), though this premise does not appear to have been tested in the literature.

Thus, the purpose of this study was to assess the effect of multidimensional biopsychosocially informed education regarding musculoskeletal conditions for insurance workers. The first aim was to gauge the effect of biopsychosocially informed education on back pain beliefs of insurance workers. The second aim was to determine if this education would influence the claims management behaviours of insurance workers. The third aim was to stratify results by years of experience in the workers compensation environment, education level and if the participants had specific health/medical training. It was envisaged this study would provide preliminary support for the efficacy of biopsychosocially informed education for non-clinical stakeholders (insurance workers) in a compensation setting.

\section{Methods}

\subsection{Study design}

A single-arm intervention study for which ethical approval was provided by Curtin University Human Research Ethics Committee (Approval Number 242/2013).

\subsection{Setting and participants}

The study participants were insurance workers employed by a single insurance company (CGU Insurance Limited) operating as an 'approved' insurer within the Western Australian workers' compensation system [31]. This system is a statutory, risk based, no fault system with state government oversight (www.workcover.wa.gov.au). Insurance workers in the context of this study consisted of Case Managers and Injury Management Advisors. Case Managers are insurance workers with responsibility for monitoring day-to day management of all aspects of a claim, including return to work, medical management and financial aspects of claims. They can have a highly mixed background in terms of level of education and work experience. Injury Management Advisors are insurance workers with a health care background who provide support to Case Managers, generally on the management of more complex claims where suboptimal return to work progress has occurred. Subjects provided written informed consent to participate in the study.

\subsection{Educational sessions and material}

Participants (both Case Managers and Injury Management Advisors) attended two, 1.5 hour education sessions held two weeks apart. The education was 
Table 1

Multidimensional biopsychosocial barriers to recovery in injured works covered in the education material

\begin{tabular}{ll}
\hline PAIN FEATURE RELATED & PSYCHOLOGICAL FACTORS \\
Very High Pain Intensity & -Cognitive \\
Radicular Pain & Low Recovery Expectations \\
Neuropathic/Neuropathic-Like Pain & Pain Beliefs \\
Widespread Pain & Fear Avoidance \\
History of Pain & Coping \\
& Passive Approach to Recovery \\
OCCUPATIONAL FACTORS & -Emotional \\
Work Demands & Depression, Anxiety and Stress \\
Work Support & Post-Traumatic Stress \\
Job Satisfaction & Anger \\
Compensation & \\
& SOCIAL FACTORS \\
PHYSICAL FACTORS & Education \\
Obesity & Income \\
Reduced Strength and Fitness (Deconditioning) & Home Support \\
Habitual Postures and Movement Patterns & \\
& \\
OTHER FACTORS & LIFESTYLE FACTORS \\
Age & Sleep \\
Poor General Health & General Activity Levels \\
Gender & Smoking \\
\hline
\end{tabular}

part of mandatory training for all employees, who subsequently all volunteered to be part of the study. The education program was developed via collaboration between the primary researchers (DB and TM) and injury management representatives of the insurance company (including NP and JW). In line with the purpose of the study, the content of the education material conformed to contemporary knowledge regarding musculoskeletal conditions and identification of barriers to recovery of injured workers with musculoskeletal conditions from an evidence based biopsychosocial perspective (Table 1). Additionally, identification of positive factors within a patient's presentation were emphasized (eg resilience, compliance, motivation). Key themes in the Day 1 material were injury taxonomy, pain taxonomy, specific versus non-specific diagnoses, diagnostic investigations, information related to interpretation of these investigation results, and the roles of different health care practitioners. Themes of the Day 2 education session were multidimensional biopsychosocial risk factors for poor recovery in injured workers, with an introduction to the use of screening tools for risk profiling and use of validated questionnaires for tracking worker progress (long and short form of the Örebro Musculoskeletal Pain Questionnaire [32], STarT Back Screening Tool [33]). Day 2 included an interactive session involving identification of risk factors. The primary researchers presented this material (DB,
TM). Participants were supplied with a summary document of the concepts covered during the education sessions for future reference https://www. painoptions.com.au/resources/useful-resources/.

\subsection{Measures}

Both Case Managers and Injury Management Advisors completed all measures except for the observational review of claims management practice, which was only completed by Injury Management Advisors. Data from these two groups were combined based on the rationale that stratification by demographic data would account for inherent differences in these two staff roles. Furthermore, there was no basis to expect different baseline beliefs between these two groups. Three types of self-reported measures were obtained, including demographic data, beliefs and attitudes regarding pain and disability, and impact of education on claims management behaviors. Demographic data were obtained prior to the first education session for age, sex, years of experience in the workers' compensation setting, educational level and whether or not the participant had specific health/medical training.

Two questionnaires were used to gauge the effect of the education on insurance workers attitudes and beliefs. The Health Care Providers' Pain and Impairment Relationship Scale (HC-PAIRS) [34] was used 
to assess insurance workers beliefs and expectations related to the functional abilities of patients with chronic low back pain [34]. Participants rated 15-items on the questionnaire using a seven-point Likert scale with prompts ranging from 'completely disagree' to 'completely agree'. Potential scores ranged from 15 to 105 , with higher scores indicating a greater belief that pain justifies decreased activity performance [35]. A difference in scores in the vicinity of half of one standard deviation has been considered clinically meaningful [26]. The Back Beliefs Questionnaire (BBQ) was used to assess beliefs related to the future course and inevitability of back pain [36]. Participants rate 14-items on a five-point Likert scale with prompts ranging from 'completely disagree' to 'completely agree'. Ratings from nine questions are reversed and provide a BBQ score that can range from 9 to 45 , with lower scores indicating more negative beliefs. A difference in scores of 2 points or more has been considered clinically meaningful $[37,38]$.

While the education was not body region specific, these two measures pertaining to back pain beliefs were chosen because of their established use in the literature and the absence of similar non-body region specific alternatives. Both the HC-PAIRS and the BBQ have established reliability and validity $[36,39$, 40] and are sensitive to change following education $[26,28,41]$.

These questionnaires were completed prior to the first education session, immediately following the second education session, and at the three-month follow-up.

Changes in claims management behaviours of insurance workers were assessed at the three-month follow-up via two methods. Firstly, assessment was made via self-reported change in practice of insurance workers using responses to the following questions: "Did the education sessions result in a change in the way you manage claims?" (11 point Likert scale, $0=$ No change at all, $10=$ Completely changed) and "Did you find the education sessions useful?" (11 point Likert scale, $0=$ Not useful at all, $10=$ Extremely useful). Secondly, Injury Management Advisors only were asked to comment on changes they had observed in interaction with their co-workers following receiving education. Specifically, the Injury Management Advisors were asked; "Have you noticed any change in practice in the case managers following the education sessions?", "Please comment on positive changes? (if none please state so)" and "Please comment on negative changes? (if none please state so)".

\subsection{Analysis}

Analysis was performed with Stata 13.1 (StataCorp, Texas, USA, Macintosh version). Demographic data were assessed with standard descriptive statistics. Demographics of participants lost to follow-up were compared to those remaining at the three-month follow-up with $t$-tests or cross-tabulation with chi-square analyses as appropriate. For the first aim, the HC-PAIRS and BBQ were compared across the three time points using repeated measure ANOVA. For the second aim, self-reported change in claims management and reported usefulness of the education sessions were reported descriptively. Comments from the Injury Management Advisers were tabulated.

For the third aim, a change in score variable for the HC-PAIRS and BBQ were created by subtracting pre-education scores from the three-month follow-up scores. Simple correlations were performed to assess associations between years of experience as an insurance worker separately with HC-PAIRS, BBQ and self-reported change in claims management. One-way ANOVAs were performed to assess for influence of educational level separately on HCPAIRS, BBQ and self-reported change in practice. $t$-tests were performed to assess for influence of a history of health/medical training separately on HC-PAIRS, BBQ and self-reported change in claims management.

\section{Results}

Forty-four insurance workers (39 case managers and 5 injury management advisors) attended the first education session. Of those, 36 also attended the second education session. Participants who did not attend the second education session were not available due to illness, leave or other work commitments, including management demands of more senior case workers. After the three-month follow-up there were full data-sets for 32 participants. Table 2 displays the demographic characteristics of the final cohort, as well as demographics for the 12 participants lost to follow-up. Those lost to follow-up tended to be older, had more experience and did not have a medical health/training background (Table 2).

In relation to the first aim, significant improvements in the scores for the HC-PAIRS and BBQ questionnaires were found post-education $(p<0.05)$ (Table 3). Specifically, scores on the HC-PAIRS 
Table 2

Baseline demographic data for participants with comparisons between the final cohort who provided data pre-education, immediately post-education and at the 3 month follow-up versus those who were lost to follow-up. Figures in table are mean (standard deviation) or number (percentage)

\begin{tabular}{|c|c|c|c|}
\hline & Final Cohort & Drop-outs & $p$ \\
\hline & $(n=32)$ & $(n=12)$ & \\
\hline Age & $32.7(11.8)$ & $41.6(11.2)$ & 0.03 \\
\hline Females & $22(69 \%)$ & $9(75 \%)$ & 0.69 \\
\hline How many years of experience do you have as an insurance worker? & $6.5(7.9)$ & $16.0(12.5)$ & $<0.001$ \\
\hline \multicolumn{4}{|l|}{ Highest level of education? } \\
\hline None & $1(3 \%)$ & $1(8 \%)$ & \\
\hline Secondary School & $11(34 \%)$ & $4(33 \%)$ & 0.77 \\
\hline Technical College & $8(25 \%)$ & $4(33 \%)$ & \\
\hline University & $12(38 \%)$ & $3(25 \%)$ & \\
\hline \multicolumn{4}{|l|}{ Did you have health/medical training prior to being an insurance worker? } \\
\hline Yes & $9(28 \%)$ & $0(0 \%)$ & \\
\hline No & $23(72 \%)$ & $12(100 \%)$ & 0.04 \\
\hline \multicolumn{4}{|l|}{ HC-PAIRS } \\
\hline Pre-education & $55.7(11.5)$ & $59.0(13.3)$ & 0.42 \\
\hline \multicolumn{4}{|l|}{ Back Beliefs Questionnaire } \\
\hline Pre-education & $33.7(5.6)$ & $35.0(4.2)$ & 0.48 \\
\hline
\end{tabular}

Table 3

Changes in the Health Care Providers' Pain and Impairment Relationship Scale (HC-PAIRS) and the Back Beliefs Questionnaire (BBQ) following biopsychosocially informed education $(n=32)$. (mean(standard deviation))

\begin{tabular}{lllll}
\hline & $\begin{array}{c}\text { Pre- } \\
\text { Education }\end{array}$ & $\begin{array}{c}\text { Post- } \\
\text { Education }\end{array}$ & $\begin{array}{c}\text { 3 Month } \\
\text { Follow-up }\end{array}$ & P \\
\hline HC-PAIRS & $55.7(11.5)$ & $50.6(12.7)^{\mathrm{a}}$ & $51.5(14.3)^{\mathrm{a}}$ & 0.009 \\
BBQ & $33.7(5.6)$ & $35.4(4.6)^{\mathrm{a}}$ & $35.7(5.7)^{\mathrm{a}}$ & 0.049 \\
\hline
\end{tabular}

${ }^{\mathrm{a}}$ Denotes significant difference from Pre-Education in post-hoc comparisons.

decreased by 5.1 points and scores on the BBQ increased by 1.7 points. These significant changes were maintained at three-month follow-up (HCPAIRS mean difference 4.2, BBQ mean difference 2.0) (Table 3). Not only were these changes statistically significant, but the magnitude of change in both measures were consistent with clinically significant changes based on previous guidelines reported above [26, 37, 38].

In relation to the second aim, Fig. 1 shows a normal distribution in terms of self-perceived change in claims management behaviours at the three-month follow-up (mean rating of 4.8, standard deviation 2.4 ), with more than $50 \%$ of participants reporting a change of 5 or more points out of 10 on a numeric rating scale. Over $80 \%$ of participants found the education sessions useful (mean rating of 7.3, standard deviation 2.1) scoring 5 or more out of 10 (Fig. 1). All five Injury Management Advisors working for the insurer independently reported observed positive changes in the behaviors of their case manager co-

\section{(A)}

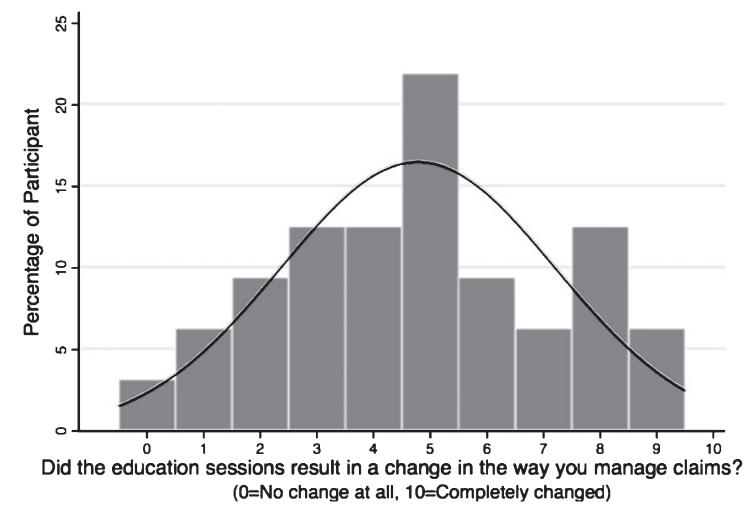

(B)

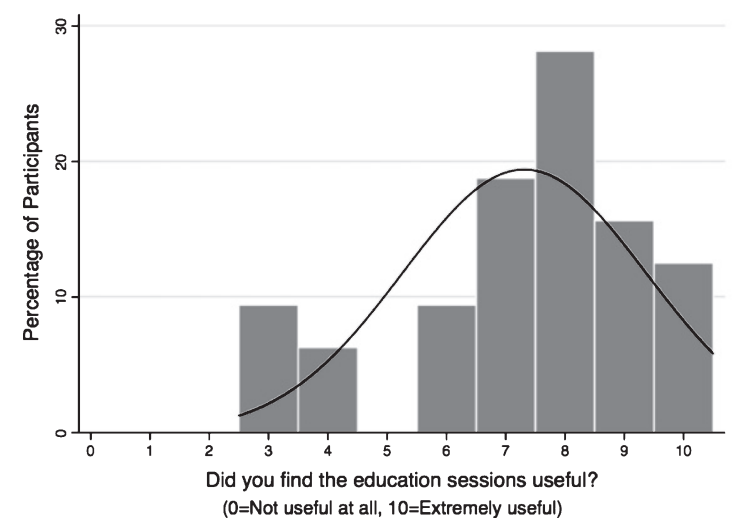

Fig. 1. Insurance workers self-perceived change in claims management behaviour (A) and perception of usefulness of the biopsychosocially informed education sessions (B) at the threemonth follow-up time point. 
Table 4

Observations of Injury Management Advisors related to positive behavioural changes in their coworkers

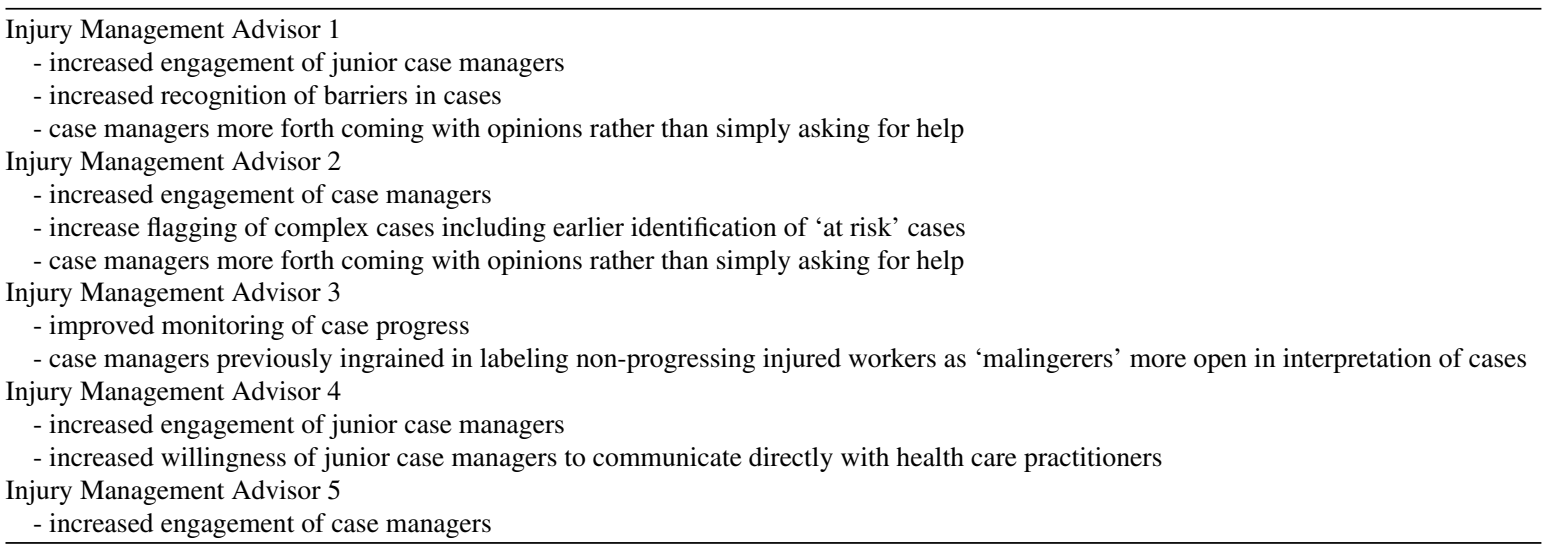

workers (Table 4). Common themes were increased engagement of case managers with Injury Management Advisors and case managers more forthcoming with seeking opinions and guidance on more complicated claims. There were no comments related to negative changes, though one Injury Management Advisor did comment that not all case managers had changed their practice.

Correlation between insurance workers years of experience with HC-PAIRS change score was $r=0.21(p=0.24)$, with BBQ change was $r=-0.20$ $(p=0.26)$ and with self reported change in practice was $r=-0.30(p=0.10)$. Table 5 shows the association of; education level and previous health/medical training on the change in the HC-PAIRS and BBQ at the three-month follow-up, and the self-rated change in claims management. The only significant finding was that individuals with past health/medical training reported a higher level of change in claims management behaviours three months after the education sessions (mean response of 6.2 (standard deviation $=1.8$ ) for those with medical training versus 4.2 (standard deviation $=2.4$ ) for those without $(p=0.03)$ ).

\section{Discussion}

The results of this study support that brief biopsychosocially informed education can improve insurance workers' back pain beliefs. Further, the education appeared to have a positive effect on the claims management behaviour of the insurance workers. Given that insurance workers are key non-clinical stakeholders in the management of injured workers, education that facilitates claim management that
Table 5

The relationship between insurance worker education level and medical training with change in the Health Care Providers' Pain and Impairment Relationship Scale (HC-PAIRS), change in the Back Beliefs Questionnaire (BBQ) and the self-reported change in claims management behaviour. Mean (standard deviation) displayed

\begin{tabular}{lll}
\hline Change in HC-PAIRS & & \\
\hline Educational Level & & \\
- Secondary School & $-4.0(10.7)$ & \\
- Technical College & $-0.9(8.4)$ & \\
- University & $6.2(15.7)$ & $p=0.65$ \\
Medical Training & & \\
- Yes & $-1.4(5.1)$ & \\
- No & $-5.2(13.8)$ & $p=0.43$ \\
Change in BBQ & & \\
Educational Level & & \\
- Secondary School & $2.5(7.2)$ & \\
- Technical College & $1.9(3.7)$ & \\
- University & $1.7(3.7)$ & $p=0.92$ \\
Medical Training & & \\
- Yes & $2.3(3.2)$ & \\
- No & $1.8(5.6)$ & $p=0.80$ \\
Self Reported Change in Practice & & \\
Educational Level & & \\
- Secondary School & $4.6(2.1)$ & \\
- Technical College & $3.9(2.8)$ & \\
- University & $5.4(2.5)$ & $p=0.39$ \\
Medical Training & & \\
- Yes & $6.2(1.8)$ & \\
- No & $4.2(2.4)$ & $p=0.03$ \\
\hline
\end{tabular}

aligns with evidence-based clinical guidelines may be a useful strategy to improve return to outcomes for injured workers.

In line with the first aim of the study, the education program demonstrated a sustained, meaningful positive shift in insurance workers' beliefs related to low back pain. Similar positive changes in back pain beliefs following biopsychosocially informed educa- 
tion have been reported in health care practitioners [25-28]. This appears to be the first time this has been demonstrated in a non-clinical population, insurance workers. Given the education was not body region specific, it is likely there was a general shift in beliefs related to all musculoskeletal conditions, although only back pain beliefs were measured.

More positive beliefs have been associated with more positive health care practitioner behaviours including advice given to patients regarding participation in activity and work [28, 30, 42-44]. As per the second aim of the study, a positive shift in changes in claims management was reported by at least half the participants. This is consistent with previous research in health care practitioners that has demonstrated not only changes in beliefs, but changes in practice behaviours following biopsychosocially informed education [28, 30]. This has been identified as an area of need for insurance workers [15]. For example, where evidence-based guidelines support a specific course of management for a musculoskeletal injury (such as low back pain), if non-clinical stakeholders are also informed regarding these guidelines, then facilitation of appropriate management early in an injured worker's claim is arguably more likely.

In complex claims or claims involving prolonged work absence, insurance workers require knowledge regarding identification and interpretation of barriers to recovery, and skills in planning how to manage these barriers [13]. This knowledge does not appear to be part of standard education or training programs for insurance workers, despite such knowledge clearly being central to the effective management of complicated claims. Insurance workers clearly found the education designed to increase knowledge in this area to be useful. This finding, coupled with positive changes in beliefs and practice, suggest pursuing educational programs of this nature could be valuable.

Although the insurance workers are only one aspect of a worker's journey through a compensation claim $[11,12]$, insurance workers act as a central person to facilitate communication between all stakeholders involved in the rehabilitation of an injured worker. A goal of the education sessions in this study was to increase biopsychosocial knowledge related to musculoskeletal injury management. Although we hope that this translated into enhanced communication of insurance workers with other stakeholders, change in communication skills/behaviours was not measured. However, self-reported feedback of the
Injury Management Advisors may indicate that communication was improved. Additional education specifically targeting communication strategies may be a useful adjunct to the education provided in this study. Furthermore, Injury Management Advisors identified increased proactive behaviours from the Case Managers. This benefit of the education aligns to the identified need for greater proactivity in workers' compensation systems [12].

In examination of the third aim, factors such as experience, education level and history of health/medical training on the whole did not have a significant effect on the outcome of the education. While the power of the study may limit the interpretation of these findings, it suggests a broad education package such as the one developed for this study can be useful for a spectrum of insurance workers rather than needing to target specific groups of insurance workers, such as those with less experience. While speculative, it would seem logical that early exposure of new insurance workers to the evidence based biopsychosocial principles of managing musculoskeletal conditions would be beneficial. The one exception may be that insurance workers without health/medical training may benefit from slightly different education approach given they had a somewhat lower self-reported level of change in claims management practice compared to their colleagues with health/medical training background. Exploration of optimal educational parameters (eg. number of sessions, lengths of sessions, time between sessions) to facilitate retention may be another area for further investigation [45].

\subsection{Strengths and limitations}

Engaging an insurer and having them allocate time for education in an environment of competing priorities can be a challenge. Collaboration at all stages, from inception through implementation and analysis was important in achieving positive outcomes and maximizing relevance for the insurer [11]. This strength of this study also allowed for a pragmatic design that facilitated translation of contemporary evidence related to barriers to recovery for injured workers.

The single-arm study design may be considered a limitation in the interpretation of the results. No direct causal relationship between changing insurance worker's beliefs and claims management behaviours can be made from this study. It could be argued that uncontrolled factors such as improving 
workplace satisfaction by allocating time within work for continuing education, rather than the education itself, may have been behind the positive outcomes. Further, this study only measured perceptions of change in behavior. However, the positive nature of the findings with this intervention support the need and rationale for further investigation using a more robust study design.

While we found a positive finding regarding a shift in claims management behaviour, as this was assessed by self-reported change, the finding does limit extrapolation. For example, we do not know what aspects of claims management changed. Further, on an individual basis, highly functioning insurance workers may not have needed to change their practice following the education. Further research might investigate the nature of practice change in more detail, particularly utilising systematic qualitative procedures.

\subsection{Conclusion}

Insurance workers' beliefs related to back pain can be positively influenced with a brief biopsychosocially informed education program. Insurance workers valued this type of education, and such education may have the potential to facilitate changes in claims management behaviours. These preliminary findings support that further research is required to ascertain if positive changes in insurance workers' beliefs and claims management behaviours result in improved return to work outcomes and decreased claims costs.

\section{Acknowledgments}

DB was supported by an Australian National Health and Medical Research Council Fellowship (APP1036778).

\section{Conflict of interest}

The authors have no conflict of interest to report.

\section{References}

[1] Australian Institute of Health and Welfare. Australia's Health 2008. Canberra, ACT: Australian Institute of Health and Welfare, 2008 Contract No.: AIHW cat.no. AUS 73.

[2] van Leeuwen MT, Blyth FM, March LM, Nicholas MK, Cousins MJ. Chronic pain and reduced work effective- ness: The hidden cost to Australian employers. Eur J Pain 2006;10(2):161-6.

[3] Walker BF, Muller R, Grant WD. Low back pain in Australian adults: The economic burden. Asia Pac J Public Health 2003;15(2):79-87.

[4] McDonald M, DiBonaventura M, Ullman S. Musculoskeletal pain in the workforce: The effects of back, arthritis, and fibromyalgia pain on quality of life and work productivity. J Occup Environ Med 2011;53(7):765-70.

[5] Woolf AD, Erwin J, March L. The need to address the burden of musculoskeletal conditions. Best Pract Res Clin Rheumatol 2012;26(2):183-224.

[6] Vos T, Flaxman AD, Naghavi M, Lozano R, Michaud C, Ezzati M, et al. Years lived with disability (YLDs) for 1160 sequelae of 289 diseases and injuries 1990-2010: A systematic analysis for the Global Burden of Disease Study 2010. Lancet 2012;380(9859):2163-96.

[7] WorkCover WA. Workers' Compensation in Western Australia Annual Statistical Report 2007/08 to 2010/11: http://www.workcover.wa.gov.au; 2012.

[8] Workcover WA. Scheme status report, December 2013. http://www.workcover.wa.gov.au: 2013.

[9] Safe Work Australia. Compendium of Workers' Compensation Statistics Australia 2009-10. Canberra: 2012.

[10] Martin BI, Turner JA, Mirza SK, Lee MJ, Comstock BA, Deyo RA. Trends in health care expenditures, utilization, and health status among US adults with spine problems, 1997-2006. Spine 2009;34(19):2077-84.

[11] Franche RL, Baril R, Shaw W, Nicholas M, Loisel P. Workplace-based return-to-work interventions: Optimizing the role of stakeholders in implementation and research. J Occup Rehabil 2005;15(4):525-42.

[12] Hallden J. The original intent of workers' compensation: A team approach. Work 2014;48(3):435-9.

[13] Linton SJ, Boersma K. Early identification of patients at risk of developing a persistent back problem: The predictive validity of the Orebro Musculoskeletal Pain Questionnaire. Clin J Pain 2003;19(2):80-6.

[14] Beales DJ, Ruscoe GA, editors. Physiotherapists and insurance workers perceptions and beliefs of roles in the West Australian Workers' Compensation System. Australian Physiotherapy Association Conference Week; 2013; Melbourne, Australia.

[15] Beales DJ, Ruscoe GA. Living in parallel universes: Physiotherapists and insurance workers' beliefs in the West Australian workers' compensation system. IAIABC Journal 2013;50(1):35-62.

[16] Salazar MK, Graham KY. Evaluation of a case management program. Summary and integration of findings. AAOHN J 1999;47(9):416-23.

[17] Pransky GS, Loisel P, Anema JR. Work disability prevention research: Current and future prospects. J Occup Rehabil 2011;21(3):287-92.

[18] Waddell G. Preventing incapacity in people with musculoskeletal disorders. Brit Med Bull 2006;77-78:55-69.

[19] Waddell G, Burton AK. Concepts of rehabilitation for the management of low back pain. Best Pract Res Clin Rheumatol 2005;19(4):655-70.

[20] Laisne F, Lecomte C, Corbiere M. Biopsychosocial determinants of work outcomes of workers with occupational injuries receiving compensation: A prospective study. Work 2013;44(2):117-32.

[21] Deeney C, O'Sullivan L. Work related psychosocial risks and musculoskeletal disorders: Potential risk factors, causation and evaluation methods. Work 2009;34(2):239-48. 
[22] Pincus T, Kent P, Bronfort G, Loisel P, Pransky G, Hartvigsen J. Twenty-five years with the biopsychosocial model of low back pain-is it time to celebrate? A report from the twelfth international forum for primary care research on low back pain. Spine 2013;38(24):2118-23.

[23] Foster NE, Delitto A. Embedding psychosocial perspectives within clinical management of low back pain: Integration of psychosocially informed management principles into physical therapist practice-challenges and opportunities. Phys Ther 2011;91(5):790-803.

[24] Darlow B, Fullen BM, Dean S, Hurley DA, Baxter GD, Dowell A. The association between health care professional attitudes and beliefs and the attitudes and beliefs, clinical management, and outcomes of patients with low back pain: A systematic review. Eur J Pain 2012;16(1):3-17.

[25] Beales D, O'Sullivan P. Beliefs of Australian physical therapists related to lumbopelvic pain following a biopsychosocial workshop. J Phys Ther Ed 2014;28(3):128-33.

[26] Domenech J, Sanchez-Zuriaga D, Segura-Orti E, EspejoTort B, Lison JF. Impact of biomedical and biopsychosocial training sessions on the attitudes, beliefs, and recommendations of health care providers about low back pain: A randomised clinical trial. Pain 2011;152(11):2557-63.

[27] O'Sullivan KJ, O'Sullivan PB, O'Sullivan L, Dankaerts W. Back pain beliefs in health care professionals are more positive after brief biopsychosocially orientated workshops. Physiotherapy Practice and Research 2012;34:37-45.

[28] Slater H, Davies SJ, Parsons R, Quintner JL, Schug SA. A policy-into-practice intervention to increase the uptake of evidence-based management of low back pain in primary care: A prospective cohort study. PLoS One 2012;7(5):e38037.

[29] Buchbinder R. Self-management education en masse: Effectiveness of the Back Pain: Don't Take It Lying Down mass media campaign. Med J Aust 2008;189(10 Suppl):S29-32.

[30] Evans DW, Breen AC, Pincus T, Sim J, Underwood M, Vogel $\mathrm{S}$, et al. The effectiveness of a posted information package on the beliefs and behavior of musculoskeletal practitioners: The UK Chiropractors, Osteopaths, and Musculoskeletal Physiotherapists Low Back Pain ManagemENT (COMPLeMENT) randomized trial. Spine 2010;35(8):858-66.

[31] WorkCover WA. Workers' Compensation in Western Australia Annual Statistical Report 2008/09 to 2011/12: http://www.workcover.wa.gov.au; 2013.

[32] Linton SJ, Nicholas M, MacDonald S. Development of a short form of the orebro musculoskeletal pain screening questionnaire. Spine 2011;36(22):1891-5.
[33] Hill JC, Dunn KM, Lewis M, Mullis R, Main CJ, Foster $\mathrm{NE}$, et al. A primary care back pain screening tool: Identifying patient subgroups for initial treatment. Arthritis Rheum 2008;59(5):632-41.

[34] Rainville J, Bagnall D, Phalen L. Health care providers' attitudes and beliefs about functional impairments and chronic back pain. Clin J Pain 1995;11(4):287-95.

[35] Ryan C, Murphy D, Clark M, Lee A. The effect of a physiotherapy education compared with a non-healthcare education on the attitudes and beliefs of students towards functioning in individuals with back pain: An observational, cross-sectional study. Physiotherapy 2010;96(2):144-50.

[36] Symonds TL, Burton AK, Tillotson KM, Main CJ. Do attitudes and beliefs influence work loss due to low back trouble? Occup Med 1996;46(1):25-32.

[37] George SZ, Teyhen DS, Wu SS, Wright AC, Dugan JL, Yang G, et al. Psychosocial education improves low back pain beliefs: Results from a cluster randomized clinical trial (NCT00373009) in a primary prevention setting. Eur Spine J 2009;18(7):1050-8.

[38] Buchbinder R, Jolley D, Wyatt M. Population based intervention to change back pain beliefs and disability: Three part evaluation. BMJ 2001;322(7301):1516-20.

[39] Bishop A, Thomas E, Foster NE. Health care practitioners' attitudes and beliefs about low back pain: A systematic search and critical review of available measurement tools. Pain 2007;132(1-2):91-101.

[40] Bostick GP, Schopflocher D, Gross DP. Validity evidence for the back beliefs questionnaire in the general population. Eur J Pain 2013;17(7):1074-81.

[41] Latimer J, Maher C, Refshauge K. The attitudes and beliefs of physiotherapy students to chronic back pain. Clin J Pain 2004;20(1):45-50.

[42] Briggs AM, Slater H, Smith AJ, Parkin-Smith GF, Watkins K, Chua J. Low back pain-related beliefs and likely practice behaviours among final-year cross-discipline health students. Eur J Pain 2013;17(5):766-75.

[43] Houben RM, Vlaeyen J, Peters M, Ostelo R, Wolters PM, Stomp-van den Berg SG. Health care providers' attitudes and beliefs towards common low back pain: Factor structure and psychometric properties of the HC-PAIRS. Clin J Pain 2004;20(1):37-44.

[44] Rainville J, Carlson N, Polatin P, Gatchel RJ, Indahl A. Exploration of physicians' recommendations for activities in chronic low back pain. Spine 2000;25(17):2210-20.

[45] Rohrer D, Pashler H. Increasing retention without increasing study time. Curr Dir Psychol Sci 2007;16(4):183-6. 